\title{
The effects of functional electrically stimulated (FES)-arm ergometry on upper limb function and resting cardiovascular outcomes in individuals with tetraplegia: A pilot study
}

\author{
Jennifer Ptasinski, Hisham Sharif, David Ditor ${ }^{\star}$ \\ Department of Kinesiology, Brock University, St. Catharines, Canada; 'Corresponding Author: dditor@brocku.ca \\ Received 10 September 2013; revised 12 October 2013; accepted 19 October 2013 \\ Copyright (C) 2013 Jennifer Ptasinski et al. This is an open access article distributed under the Creative Commons Attribution License, \\ which permits unrestricted use, distribution, and reproduction in any medium, provided the original work is properly cited.
}

\section{ABSTRACT}

Background: Functional electrically stimulated (FES)-arm ergometry has been shown to increase peak power output and aerobic capacity in individuals with cervical $\mathrm{SCl}$. However, the functional benefits remain unknown. Objective: To determine the effects of FES-arm ergometry on exercise performance, upper limb function and resting cardiovascular function in individuals with tetraplegia. Methods: Five individuals (43.8 \pm 15.4 years old) with SCI (C3-C5, AIS C-D, $14.0 \pm 11.1$ years post-injury) completed 12 weeks FES-arm ergometry. Exercise performance (time and distance to fatigue), perceived upper limb function [Capabilities of Upper Extremity Questionnaire (CUE), short form-Quadriplegia Index of Function Questionnaire (sf-QIF) and Spinal Cord Injury Spasticity Evaluation Tool (SCI-SET)] and resting mean arterial pressure (MAP) and heart rate (HR) were measured pre and post. Results: Following training, MAP significantly decreased $(91.1 \pm 14.0$ to $87.7 \pm 14.7 \mathrm{mmHg} ; p=$ 0.04 ), and there was a trend for an increased time to fatigue $(804.6 \pm 359.4$ to $1483.8 \pm 1110.2$ sec; $p=0.08)$, distance to fatigue $(3508.4 \pm$ 3524.5 to $7412.6 \pm 7773.1 \mathrm{~m}, \mathrm{p}=0.08$ ) and the CUE scores pertaining to hand function (31.6 \pm 12.8 to $38.0 \pm 17.7 ; p=0.07$ ). Conclusion: Twelveweek FES-arm ergometry was associated with decreased resting MAP in individuals with tetraplegia, and may show promise as a means to increase exercise performance and hand function. Further research is required to verify these preliminary findings.
Keywords: Exercise; Spinal Cord Injury; Exercise Performance; Arm Function; Blood Pressure

\section{INTRODUCTION}

For individuals with cervical SCI, reductions in hand and upper limb function can be particularly debilitating. In a study conducted by Anderson, 681 individuals with SCI were surveyed, and for those with tetraplegia, the return of arm and hand function was ranked as the highest therapeutic priority [1]. This is understandable as recovery of even partial hand and arm function could potentially have a great impact on independence. Anderson also found that $96.5 \%$ of individuals with a SCI considered exercise to be important for functional recovery, however, only $56.9 \%$ had access to exercise, and only $12.2 \%$ had access to a trained therapist. These findings demonstrate a need for therapies which are targeted at upper limb and hand musculature, and have the potential to improve function and independence.

Manual arm ergometry has been shown to result in physical improvements in individuals with SCI [2]. Unfortunately, the potential benefits attained from this form of exercise are limited to those with at least partial upper limb mobility. However, functional electrically stimulated (FES) exercise is a therapeutic option for individuals with SCI and FES-arm ergometry which has recently become available. This form of exercise involves electrical stimulation of the upper limb musculature, which is in such a sequence as to cause a fluid armcycling motion. A recent study found that 12 weeks of thrice-weekly progressive FES-arm ergometry resulted in increased peak power output and peak aerobic capacity in individuals with cervical SCI [3]. Although these results are very encouraging, the functional benefits of 
FES-arm ergometry have yet to be fully determined.

Therefore, the purpose of the present study was to investigate the effects of a 12-week, thrice-weekly, FESarm ergometry program on exercise performance, upper limb function and resting cardiovascular outcomes in individuals with chronic incomplete tetraplegia. We hypothesized that 12 weeks of FES-arm ergometry would improve participants' exercise performance, upper limb function and resting cardiovascular measures.

\section{MATERIALS AND METHODS}

\subsection{Participants}

Five individuals ( 4 male, 1 female; $43.8 \pm 15.4$ years old) with chronic, incomplete SCI (C3-C5, AIS C-D, $14.0 \pm 11.1$ years post-injury) were recruited to participate in this study. Participant characteristics are detailed in Table 1. All participants received medical approval before enrolment into the study, and no participant had undertaken FES-exercise training, or any other structured exercise training, in the three months prior to the study. Individuals were excluded from the study if they had documented cardiovascular disease, a tracheostomy, uncontrolled autonomic dysreflexia, or any other medical condition contraindicating exercise. Participants were not required to discontinue their medications during the testing or training sessions, and no participants made any change in their medications during the course of training. This study was approved by the Research Ethics Board of Brock University and all participants provided informed and signed consent before participating. All procedures conformed with The Code Ethics of the World Medical Association (Declaration of Helsinki) of July 1964.

\subsection{Intervention}

FES-arm ergometry was completed on the RT300 ergometer (Restorative Therapies; Baltimore, MD), with surface electrodes $(2 \times 3.5$ inch; PALS Platinum $)$ being placed on the biceps, triceps, medial deltoids, and supraspinatus muscles. For participants with inadequate grip strength, tensor bandages were used to secure the hands

Table 1. Participant characteristics.

\begin{tabular}{cccccc}
\hline Participant & Age & Sex & Level of Injury & AIS Years Post Injury \\
\hline 1 & 26 & Male & C4 & C & 7 \\
2 & 46 & Male & C5 & C & 20 \\
3 & 30 & Male & C5 & C & 11 \\
4 & 58 & Female & C3 & D & 30 \\
5 & 59 & Male & C3 & C & 2 \\
\hline
\end{tabular}

AIS: ASIA impairment scale, ASIA denotes American Spinal Injury Association. to the grips of the RT300. The training protocol included 12 weeks of FES-arm cycling, at a frequency of three sessions per week, with a minimum of 30 minutes and a maximum of 45 minutes of active exercise per session. There was 48 hours of rest between exercise sessions whenever possible. The RT300 includes a motor that can be used to assist the pedaling motion or provide resistance as participants progress over time. Each exercise session began with a two minute passive, motor-driven warm up, followed by 30 - 45 minutes of active FES-arm ergometry, and was completed with a two minute passive, motor-driven cool down. The stimulation pulse width was set at $250 \mu \mathrm{s}$, and the stimulation frequency was set at $50 \mathrm{~Hz}$ for the biceps and triceps electrodes, and $25 \mathrm{~Hz}$ for the deltoid and supraspinatus electrodes. The stimulation current amplitude was adjustable between 0 to 140 $\mathrm{mA}$, however, for the present study the current was set, as individually tolerated by comfort, between 15 and 50 $\mathrm{mA}$.

The exercise sessions were progressed as individually tolerated by increasing the duration of exercise, followed by increasing the resistance provided by the motor of the arm ergometer. Specifically, each exercise session was to include at least 30 minutes of active exercise, and no more than 45 minutes of exercise. If the upper limbs fatigued before 30 minutes, then a 5 minute rest period was allowed and a subsequent bout would be performed. This would continue until at least 30 minutes of exercise had been accomplished. When a participant was able to perform two consecutive sessions of 30 minutes continuously, the resistance would be increased by one unit as indicated on the RT300. The RT300 stimulates the muscles such that a target cadence of 50 revolutions per minute is maintained, and fatigue was determined automatically by the RT300 when, despite maximal stimulation, the cadence of pedaling dropped below 35 revolutions per minute for 2 seconds. Although no maximum cadence was set, the revolutions per minute did not exceed 55 .

A maximum of five bouts per session were allotted to complete the 30 - 45 minutes of exercise per session. Participants had to maintain an exercise adherence of at least $75 \%$ for their data to be included in the analysis.

\subsection{Outcome Measures}

Exercise performance measures were collected via an exercise to fatigue test. This test was conducted at baseline and again following the completion of the 12-week training program (48 hours following the final training session), and the resistance used for each individual was held constant for each test. These measures of performance included time to fatigue and distance to fatigue.

Functional outcomes included self-reported measures of upper extremity function and independence as deter- 
mined by the Capabilities of Upper Extremity Questionnaire (CUE) [4], and the short form of the Quadriplegia Index of Function Questionnaire (sf-QIF) [5]. The CUE is a 32-item questionnaire that evaluates how well an individual can perform movements with his or her arms and hands. The left and right limbs are scored separately on a seven point scale with $1=$ totally limited and $7=$ not at all limited. The sf-QIF is a 6-item questionnaire that evaluates an individual's level of independence when performing activities of daily living that require upper limb function. Items are rated on a 5-point scale with $0=$ dependent and $4=$ independent.

Self-reported muscle spasticity was determined by the Spinal Cord Injury Spasticity Evaluation Tool (SCI-SET) [6]. This 35-item, 7-day recall questionnaire does not evaluate the amount of spasticity per se, but rather, it evaluates how spasticity affects (either positively or negatively), certain activities of daily living. The SCI-SET questionnaire employs a 7-point scale with $-3=$ extremely problematic, $0=$ no effect, and $3=$ extremely helpful. All functional outcome measures were made at baseline and following the 12-week exercise training program.

Resting mean arterial blood pressure (MAP) and heart rate (HR) were conducted at baseline and following twelve weeks of FES-arm ergometry exercise (48 hours following the final training session). Resting cardiovascular measures were conducted in the supine position following 10 minutes of rest in a dark, quiet room. MAP and HR were determined via an automated cuff placed over the left brachial artery, and measures were taken twice per test and averaged to determine the true resting value.

\subsection{Statistical Analysis}

All statistical analyses were conducted with the Statistica software program. Due to the relatively small sample size and high amount of baseline variability between participants, outcome measures were compared pre and post-testing with non-parametric statistical analysis. Specifically, the Wilcoxon test was used to compare means at pre and post-testing. Effect size (ES) calculations were also conducted on all outcome measures for the baseline and 12 -week values. Statistical significance was set at $\mathrm{p}$ $<0.05$, and all values are expressed as mean \pm standard deviation (SD).

\section{RESULTS}

Participants completed the 12-week exercise training program, without any adverse events or exercise-induced injury. The average adherence rate was $85 \%$, with adherence being defined as the percentage of scheduled sessions attended and completed.

\subsection{Exercise Performance}

Following the 12-week exercise program, there were no significant changes in exercise performance, however, there were trends towards improvement in both time to fatigue $(804.6 \pm 359.4$ to $1483.8 \pm 1110.2 \mathrm{sec} ; \mathrm{p}=0.08$; $\mathrm{ES}=1.14)$ and distance to fatigue $(3508.4 \pm 3524.5$ to $7412.6 \pm 7773.1 \mathrm{~m}, \mathrm{p}=0.08 ; \mathrm{ES}=1.24)$ during the exercise performance test (Table 2).

\subsection{Functional Outcomes}

Following the 12-week exercise program, there was no significant change in the composite score for the CUE (Table 3). Likewise, when considering the subscales of the CUE, there was no significant change in shoulder, biceps, wrist extensor, triceps, or trunk function (Table 3). However, there was a trend towards an increase in the CUE subscale pertaining to hand function following the 12-week exercise program (Table 3 ). There was no significant change in upper limb function as determined by the sf-QIF, or the SCI-SET, following the training program (Table 3).

Table 2. Measures of exercise performance before and after 12 weeks of FES-arm ergometry.

\begin{tabular}{ccccc}
\hline & Pre & Post & p-value & ES \\
\hline $\begin{array}{c}\text { Time to } \\
\text { fatigue (sec) }\end{array}$ & $804.6 \pm 359.4$ & $1483.8 \pm 1110.2$ & 0.08 & 1.14 \\
$\begin{array}{c}\text { Distance to } \\
\text { fatigue (m) }\end{array}$ & $3508.4 \pm 3524.5$ & $7412.6 \pm 7773.1$ & 0.08 & 1.24 \\
\hline ES: Effect Size. & & & &
\end{tabular}

Table 3. Measures of upper limb function before and after 12 weeks of FES-arm ergometry.

\begin{tabular}{ccccc}
\hline Scale & Pre & Post & p-value & ES \\
\hline CUE (Composite) & $119.6 \pm 49.4$ & $127.6 \pm 54.2$ & 0.58 & 0.31
\end{tabular}

CUE (Subsets)

$\begin{array}{ccccc}\text { Shoulders } & 20.4 \pm 11.1 & 22.2 \pm 9.6 & 0.67 & 0.25 \\ \text { Biceps } & 21.0 \pm 8.2 & 18.8 \pm 8.1 & 0.35 & 0.48 \\ \text { Wrist Extensors } & 19.2 \pm 9.7 & 18.4 \pm 9.5 & 0.78 & 0.21 \\ \text { Triceps } & 23.8 \pm 10.1 & 23.0 \pm 9.9 & 0.58 & 0.26 \\ \text { Hands } & 31.6 \pm 12.8 & 38.0 \pm 17.7 & 0.07 & 1.16 \\ \text { Trunk } & 5.2 \pm 3.6 & 6.4 \pm 5.0 & 0.18 & 1.01 \\ \text { sf-QIF } & 7.8 \pm 9.8 & 9.2 \pm 11.0 & 0.2 & 0.72 \\ \text { SCI-SET } & 122.6 \pm 14.9 & 129.8 \pm 9.2 & 0.23 & 0.72\end{array}$

CUE: Capabilities of Upper Extremity Questionnaire; sf-QIF: short formQuadriplegia Index of Function Questionnaire; SCI-SET: Spinal Cord Injury Spasticity Evaluation Tool; ES: Effect Size. 


\subsection{Cardiovascular Outcomes}

There was a statistically significant decrease in resting MAP following the 12-week FES-arm ergometry program $(91.1 \pm 14.0$ to $87.7 \pm 14.7 \mathrm{mmHg} ; \mathrm{p}=0.04 ; \mathrm{ES}=$ $1.63)$, but there was no change in resting HR $(66.8 \pm 7.9$ to $70.0 \pm 10.1$ beats $/ \mathrm{min} ; \mathrm{p}=0.14 ; \mathrm{ES}=1.06$ ) (Table 4).

\section{DISCUSSION}

The current pilot study was preliminary in nature, and thus the results should be interpreted with caution. Still, the main finding of the current study was that individuals with incomplete tetraplegia may experience decreases in resting MAP following 12 weeks of FES-arm ergometry, and although only trends were detected, this form of therapy may also show promise for improving exercise performance and self-reported hand function. Improvements in exercise performance and hand function would have obvious practical benefit. However, whether or not the observed cardiovascular effects are clinically significant is questionable. Individuals with SCI do experience higher rates of cardiovascular disease, however, our participants were normotensive both before and after the training program and the magnitude of the reduction in MAP was not profound. Still, this finding does hold promise for cardiovascular improvement following FES-arm exercise and research in hypertensive participants with SCI is warranted.

\subsection{Exercise Performance}

As mentioned, there were no significant changes in exercise performance in the present study, although there were trends for an increased time and distance to fatigue. In related work, Coupaud and colleagues conducted a pilot study investigating the effects of FES-arm ergometry on two individuals with tetraplegia [3]. In that pilot work, the authors found variable results on exercise performance that seemed to depend on functional ability. Specifically, their more able participant (C6, AIS B, 18 years post-injury) showed increases in peak oxygen uptake $(0.7$ to $1.1 \mathrm{~L} / \mathrm{min})$ and peak power output (7 to 38 Watts) following 12 weeks of progressive FES-arm ergometry, while their less able participant (C6, AIS A, 8 months post-injury) made no improvement in peak oxygen uptake and a smaller increase in peak power output

Table 4. Cardiovascular outcomes before and after 12 weeks of FES-arm ergometry.

\begin{tabular}{ccccc}
\hline & Pre & Post & p-value & ES \\
\hline MAP (mmHg) & $91.1 \pm 14.0$ & $87.7 \pm 14.7$ & 0.04 & 1.63 \\
HR (beats/min) & $66.8 \pm 7.9$ & $70.0 \pm 10.1$ & 0.14 & 1.06 \\
\hline
\end{tabular}

ES: Effect Size; HR: Heart Rate; MAP: Mean Arterial Pressure.
( 3 to 8 Watts). The present study lacked the statistical power to formally assess correlations between baseline function and exercise-induced improvement. However, anecdotally, our two most able participants did experience large improvements in exercise performance $(289 \%$ and $87 \%$ in time to fatigue; $336 \%$ and $78 \%$ in distance to fatigue), while the others made little to no change. Further research with larger samples is required to determine the relationship between baseline function and the potential for benefit following FES-arm ergometry.

\subsection{Upper Limb Function}

There were no significant changes in the CUE when considering the composite score for the group as a whole, however, analyzing the separate subscales yielded more encouraging results. Most notably, there was a trend for an increase in hand function following the 12-week FESarm ergometry program, and these improvements were observed in 4 of the 5 participants, while one participant showed no change. This was an interesting finding, especially given that the biceps, triceps and shoulders were electrically stimulated and not the hands or forearms. Anecdotally, spinal cord injured individuals in our rehabilitation center, who did not participate in this study, also reported improved hand function after using FESarm ergometry for their weekly therapy. It is not clear why hand function improves despite no stimulation of the hands or forearms. However, stimulating and strengthening the shoulders, biceps and triceps, may increase upper limb stability while performing tasks that involve the hands. Regarding the range of perceived benefits for hand function, they seemed to encompass a fairly wide array of gross and fine motor abilities, as the questions pertained to a wide array of tasks. Further research is certainly warranted to determine who may yield clinically important benefits in hand function following FESarm ergometry, and what the optimal training stimuli are to realize these results. Unfortunately, however, there were no significant changes in biceps, triceps or shoulder function, and this finding was surprising since those are the muscles stimulated during the FES-arm ergometry. Still, the lack of significant results for these data may be due, in part, to the ceiling effect inherent in the CUE questionnaire, as three of our participants had relatively high baseline scores for the biceps and triceps. Nonetheless, there were participants with room to improve who still showed no benefit in biceps or triceps function, and further research is required to determine who may yield upper limb benefit from FES-arm ergometry and to what degree. There were also no significant changes on the sfQIF following the 12-week training program. This lack of improvement may be due in small part to the ceiling effect inherent in the questionnaire, as one participant 
was either independent, or independent with devices, in all 6 items at baseline. Still, consistent with the results of the CUE, there were participants with room to improve on the sf-QIF who showed no benefit following the training. Furthermore, the lack of functional improvements could be due to the nature of the exercise program, as it was not intense enough to elicit functional improvements. Training with FES at a continuous moderate intensity increases cardiovascular function, as shown previously by improvements in total aerobic capacity [3] and MAP in the present study, but clearly, not functional tasks. Accordingly, our lab is currently experimenting with high intensity FES training modes as a means for potentially improving functional outcomes. Regarding the SCI-SET scores, there were no significant changes for the group as a whole, however, three of our five participants did experience moderate increases. This finding is interesting as it illustrates that any functional improvements that may ultimately be demonstrated with larger trials may be due to increases in strength and muscular endurance, or alternatively, decreases in muscle spasticity; or possibly a combination of all three factors. Other studies with larger sample sizes and a focus on strength, spasticity and function will be required to answer these questions.

\subsection{Study Limitations}

The main limitation of the current study was the small sample size. However, significant findings and some very large effect sizes were still obtained. The other notable limitation was that function was only determined by self-reported questionnaire. This type of data collection is advantageous as it represents real world function. However, the particular questionnaires that were used in the current study were susceptible to ceiling effects. As such, it is possible that our more physically capable participants, made functional gains that were not captured by the testing methods employed. Further, as questionnaires were used as a measure of upper limb function, it was perceived function that was specifically evaluated, rather than actual function demonstrated in the lab. Therefore, future studies with larger sample sizes, more invasive measures of strength or muscle morphology, and possibly a qualitative measure of function are warranted to fully understand the potential benefits of FESarm ergometry.

\section{CONCLUSION}

The current study demonstrated that individuals with incomplete tetraplegia may experience positive changes in resting blood pressure following 12 weeks of FES-arm ergometry. In addition, although only trends were found, our data justify further studies examining the potential benefits of FES-arm ergometry on exercise performance and upper limb function, and which individuals are most likely to realize such benefits.

\section{ACKNOWLEDGEMENTS}

The authors would like to acknowledge the participants of this study for their efforts and dedication. H. Sharif was supported by the Ontario Neurotrauma Foundation.

\section{REFERENCES}

[1] Anderson, K.D. (2004) Targeting recovery: Priorities of the spinal cord-injured population. Journal of Neurotrauma, 21, 1371-1383.

http://dx.doi.org/10.1089/neu.2004.21.1371

[2] Hicks, A.L., Martin Ginis, K.A., Pelletier, C.A., Ditor, D.S., Foulon, B. and Wolfe, D.L. (2011) The effects of exercise training on physical capacity, strength, body composition and functional performance among adults with spinal cord injury: A systematic review. Spinal Cord, 49, 1103-1127. http://dx.doi.org/10.1038/sc.2011.62

[3] Coupaud, S., Gollee, H., Hunt, K.J., Fraser, M.H., Allan, D.B. and McLean, A.N. (2008) Arm-cranking exercise assisted by functional electrical stimulation in $\mathrm{C} 6$ tetraplegia: A pilot study. Technology and Health Care, 16, 415-427.

[4] Marino, R.J., Shea, J.A. and Stineman, M.G. (1998) The capabilities of upper extremity instrument: Reliability and validity of a measure of functional limitation in tetraplegia. Archives of Physical Medicine and Rehabilitation, 79, 1512-1521. http://dx.doi.org/10.1016/S0003-9993(98)90412-9

[5] Marino, R.J. and Goin, J.E. (1999) Development of a short-form quadriplegia index of function scale. Spinal Cord, 37, 289-296. http://dx.doi.org/10.1038/sj.sc.3100772

[6] Adams, M.M., Martin Ginis, K.A. and Hicks, A.L. (2007) The spinal cord injury spasticity evaluation tool: Development and evaluation. Archives of Physical Medicine and Rehabilitation, 88, 1185-1192. http://dx.doi.org/10.1016/j.apmr.2007.06.012 